\title{
Parents' Personality and Their Expectations about Child's Development
}

\author{
Taslima Begum ${ }^{1, *}$, Akibul-Ul-Huque ${ }^{2}$ \\ ${ }^{1}$ BRAC Institute of Educational Development, BRAC University, Bangladesh \\ ${ }^{2}$ Department of Psychology, University of Dhaka, Bangladesh
}

Copyright $\bigcirc 2016$ by authors, all rights reserved. Authors agree that this article remains permanently open access under the terms of the Creative Commons Attribution License 4.0 international License

\begin{abstract}
The objective of the present study was to investigate the effects of parent's personality on their expectations about the development of their children. It was hypothesized that Big-five personality would predict parental expectations. Data were collected from 64 mothers $\left(X_{\mathrm{Age}}=32.76, S D=6.95\right)$ and 36 fathers $\left(X_{\mathrm{Age}}=40.08, S D\right.$ $=8.35$ ) using parental expectation questionnaire of child's development and Revised bangle version of Saucer's Big Five- Mini Markers. Multiple regression analysis indicated that openness to experience was the strongest predictor $(\beta$ $=.404, p<.05)$ followed by extraversion $(\beta=-.351, p<.05)$. The model explained $24.1 \%$ variance of parental expectations of child's development. However, agreeableness, emotional stability and conscientiousness did not predict parental expectations. Possible explanations have been put forth to account for the findings.
\end{abstract}

Keywords Parental Expectation, Personality, Child Development, Big Five Personality, Multiple Regression

\section{Introduction}

In recent decades parents demand or expect more from their children which beyond their child's ability. The effect of this kind of abuse is detrimental to the child's health, survival, and development. Its effect can become more severe as a child grows older, and encompasses multiple areas including health, physical development, emotional, cognitive and behavioral and psychological development. According to Stoiber and Houghton [1] serious attention has been given in recent years to parental expectations because they are considered to promote negative emotion toward parenting. Moreover, several researchers have indicated that parents who believe the child's behavior is inconsistent with parental goals or expectations are more likely to become abusive to their child $[2,3]$. As a result, some studies have investigated parental expectations about some areas of a child's life (e.g., academic achievement, developmental outcome, psychological outcome). As well as, some studies have conducted to investigate the detrimental of parental expectation. Among these studies McArdle and Duda [4] found parenting style and parent's self-esteem; Stoibier and Houghton [1] have found parents cognition; Flouri and Hawkes [5] have found parent's depressed mood-trait neuroticism, Beutel and Adreson [6] have found culture are associated with parental expectation. Whether self-esteem, cognition, and neuroticism are components of personality; culture and race are determinants of personality. In that situation, parents' personality might have effect on their expectation about child development. However, these studies are limited in their capacity because they do not represent overall parental expectation, rather they are about specific life domains of a child.

Although, studies have shown that parental expectations varies in different culture, race, and ethnicity [6], research about it is not conducted in Bangladesh. In addition, it is not clear to us how parental personality is related to their emerging expectation in Bangladesh. Thus we designed a study to investigate whether parents' expectations of child's development depended on their Big-five personality traits.

\subsection{Parental Expectation}

Parental expectation is the act or an instance of expectation or looking forward or hoped for something or event from their child. Hence, parental expectation is a subjective term arise from their cognition and seen as social psychological variable [7]. Parents around the world share three major goals: survival goal, economic goal and cultural goals [8]; that lead them to expect something from their children. Though, parental expectation begins while a mother is pregnant but parents shape their expectations when the child is able to do specific activities. According to Zahan [9], "Parent expectations of their children's success in life are not only an expression of their perception of the world around them but also an expression of their assessment of their ability to supervise and invest in the future their children (P. 972)". But parents often expect their 
children to be perfect and expect so much from their children that the children don't feel comfortable in their own shoes. These extreme levels of expectation are called demand.

\subsection{Personality}

Personality can be defined as consistent; unique and organized set of characteristics of a person; determined by environmental (e.g., culture, social class, family) and genetic factor that influences his or her cognition, motivation and behaviors in various situations [10]. Evidence is accumulating which suggests that virtually all personality measures can be reduced or categorized under the umbrella of a 5- factor model of personality, which has subsequently been labeled the" Big Five" [11]. The dimensionality of the Big Five has been found to generalize across virtually all cultures and remains fairly stable over time [12]. The dimensions composing the 5-factor model are neuroticism, extraversion, openness to experience, agreeableness, and Conscientiousness.

Neuroticism is a tendency to experience negative emotions, such as anger, anxiety, or depression. It is sometimes called emotional instability. Extroversion is characterized by positive emotions, and the tendency to seek out stimulation and the company of others. Openness to experiences is the trait distinguishes imaginative people from down-to-earth, conventional people. Agreeableness is a tendency to be compensate and cooperative rather than suspicious and antagonistic towards others. Conscientiousness is the trait shows a preference for planned rather than spontaneous behavior.

\subsection{Correlates of Parental Expectation and Personality}

As Personality refers to individual differences in characteristic patterns of thinking, feeling and behaving and personality traits influence people's behavior and lives in relatively stable, predictable, and meaningful ways [13] thus, assumed to have effect on parental expectation. Many studies has found correlation between personality and emotion, independence of judgment, decision making [14], creative task performance, emotional intelligence [15], and learning approach [16]. Furthermore, personality trait openness are related to media performance [17], training proficiency, learning approach toward positive attitude. Ekehammar and Akrami [18] have shown openness and agreeableness is related to prejudice. [19] has found, personality dispositions such as extraversion, neuroticism, and self-esteem can markedly influence levels of SWB people's emotional and cognitive evaluations of their lives, includes what lay people call happiness, peace, fulfillment, and life satisfaction. Extraversion and neuroticism positively related with happiness [20]. Moreover, agreeableness and openness are correlated to close relationship and marital satisfaction [21]. According to Goldberg [11], marital quality was not simply a proxy for parental personality. Parents in more satisfying and supportive marriages viewed their children more favorably and set higher standards for their children. Parental consensus about children's problem behaviors was greater when parents were martially satisfied, perceived their spouses to be supportive, and shared involvement in child care.

Where personality is said sum total of our behavior, there are some personal factor that leads parental expectation toward children. Parents' belief, knowledge, cognition, schema, judgment, child rearing belief, parent child interaction [1], dimensions of home environment [9] and other parenting behavior and pattern [22] have impact on parental expectation. Hence, nature and degrees of expectation among parents varies. McArdle and Duda's [4] study showed, parental expectation were positively related to parent's personal standard only and parent's self-esteem was predicted by high perceived parental expectation. Conversely, maternal depressed mood is associated with both low maternal expectations [23] and low maternal expectations for children's coping are related to overprotective parenting [24].

Research on parenting style, has evidenced that authoritarian and authoritative both style imposed demand on children, but demand of authoritative promote competence more than regulation of behavior [25]. Belsky \& Barends; Cumberland-Li, Eisenberg, Champion, Gershoff, \& Fabes; Losoya et al's studies (as cited in Kochanska et al.)[26] found that parental neuroticism and negative emotionality resulted in less positive, responsive, and adaptive parenting. In contrast, parental conscientiousness and agreeableness have been linked to more positive and adaptive parenting. On the other hand, findings regarding extraversion have been mixed. Extraverted parents have been seen as upbeat and engaged [27] and as endorsing nurturing, supportive parenting [28, 29], but found that highly extraverted mothers were power assertive with their toddlers [30].

Lee-Baggley, Preece, and DeLongis [31], mentioned personality plays an important role in almost every aspect of the stress and coping process. Personality has been linked to the likelihood of experiencing stressful situations, the appraisal of an event as stressful, the likelihood of engaging in certain coping strategies, and the effectiveness or outcomes of these coping strategies. However, mothers who were more conscientious tracked their children more consistently, but the overall effect of maternal personality was marginal. The infant's temperament and the father's personality each explained a marginally significant amount of variance; the overall equation was significant. Fathers' extraversion was associated with less consistent tracking. As expected, mothers high on neuroticism correlated a less positive affective ambience with their infants, consistent with a large literature on maternal depression [32, 33, 34]. It was interesting; however, that maternal Neuroticism was unrelated to responsiveness or tracking, consistent with our earlier work [30]. Perhaps a mother's ability to maintain a 
positive emotional ambience is particularly easily undermined by her elevated negative emotionality.

Belsky and Barends; Clark, Kochanska, and Ready; Goodman and Gotlib; Kochanska, Clark, and Goldman (as cited in Kochanska et al.,) [26], have found no significant links between infants' temperament and their fathers' personality traits. There were a few modest correlations between mothers' and infants' traits. More agreeable mothers had infants who were more able to focus attention, and more fearful. More empathic mothers' infants were better focused, and less prone to anger. The infants of mothers higher on Openness were more joyful.

\subsection{Hypothesis}

Based on literature review and objective of the study the following hypotheses have been formulated (1) Variation in parents' expectations of child development would be predicted by (i.e., positively related to) emotional stability, conscientiousness, openness to experience, and agreeableness. (2) Variation in parents' expectations of child development would be predicted by (i.e. negatively related to) extraversion.

\section{Method}

In the present study, data were collected from 100 parents' from Dhaka, Noakhali, and Comilla districts of Bangladesh, who have one or more children, aged above three years. Among them 64 were mothers (64\%) and $36(36 \%)$ were fathers. They were selected using multi-stage sampling (purposive and convenience sampling). Respondents' age ranged from 23 to 62, mean age was 35.39 years $(S D=8.24)$. Mothers' were aged from 23 to $57(M=32.76, S D=6.95)$ and fathers' were aged from 26 to $62(M=40.08, S D=$ $8.35)$.The monthly expanse of the participants' were ranged from 3000 to 250000 taka (mean was 28740.00 taka and $S D$ $=26151.316$ ). Education span was ranged between 0 to 25 and mean education span was14.65 $(S D=4.16)$. Mean education span of mothers' was $14.56(S D=3.36)$. Mean education span of fathers' was $14.78(S D=5.32)$. In present study, education span is defined as the total number of years one has spent in educational institutions for formal degree. The participants were selected from different occupational background with different designations, such as homemakers, teachers, doctors, professionals, businessmen etc.

\subsection{Measures}

\subsubsection{Parental Expectations Questionnaire}

Parental expectations questionnaire was developed to collect data on parental expectations about child's development. In this questionnaire parents pointed out five most important areas that they think important for the child. Then they marked the magnitude of their expectations of the child's development in these areas using 11 points where 0 indicated that they would absolutely accept the failure of the child to fulfill the expectation and 10 indicated that they would never accept the failure. At this questionnaire highest value 10 indicated parents' demand about child development.

The responded were asked to encircle one response from 11 alternatives which best described the way they had been feeling about their child's competence. The lowest possible total score obtained by someone was 0 and highest was 50 . Then average score was calculated to represent one's level of allowance.

\subsubsection{Revised Bengali Version (Huque, 2008) of Saucer's} (1994) Big Five- Mini Markers

Bangali version [35] of Saucer's (1994) Big Five- Mini Markers was revised to make some items comprehensible and simple. This scale was used to asses Big Five personality dimensions of the respondents according to their present self-perception about themselves. The scale was composed of 40 items; eight items for each factors of Big Five namely Extraversion, Agreeableness, Conscientiousness, Neuroticism, and Openness to experience. Among 40 items 20 items are negative items and 20 items are positive items. Among 20 negative items, 4 are for extraversion, 4 are for agreeableness, 4 are for conscientiousness, 2 are for openness to experience and 6 are for neuroticism.

This was a 9 point rating scale ranging from 1 to 9 where 4 items was used to indicate accurate and another 4 items was used to indicate inaccurate. Among them 1 indicatedextremely inaccurate, 2 indicated- very inaccurate, 3 indicated-moderately inaccurate, 4 indicated- slightly inaccurate, 5 indicated- neither inaccurate nor accurate, 6 indicated- slightly accurate, 7 indicated- moderately accurate, 8 indicated-very accurate, 9 indicated- extremely accurate. The total score in any factor ranged between 8 and 72 with higher score indicating predominance of the factor in the personality makeup of the individual.

Saucer [36] estimated internal consistency for the English Version five Mini-Marker scales in four data sets. The 20 Alpha coefficients ranged from .69 to .86 , averaging around .80. Research demonstrated that the Mini-Makers had some level of concurrent validity with other questionnaire based assessments of Big Five (e.g., [37]). Mullins, Polson, Lanch, and Kehoc [38] further found support for the construct validity of the measure.

To determine the translation reliability of the Bengali version Mini-Markers, both Bengali and English version Mini-Markers were administered to 50 students of University of Dhaka. Half of the respondents were given the English version first. And then after three weeks they were given the Bengali version. The correlation between the respondents to Bengali and English version Mini-Markers was highly significant $\left(r_{48}=.911, p<.01\right)$. The high Alpha coefficients $(\alpha=.567)$ indicated internal consistency of the Bengali version scale. 


\subsection{Procedure}

Data were collected individually from each respondent. Participants were given written instructions printed within the questionnaires and necessary verbal instructions about their tasks. Some respondents filled in the questionnaire themselves, but most participants were asked the question verbally and the responses were recorder accordingly. In both case, the questions were given with further clarifications whenever necessary. Further, to enhance their understanding of the meaning, it was required to give them relevant real-life because of complicated nature of some questions. In every circumstance standardized procedure was tried to follow to collect data.

\subsection{Data Analysis}

Pearson correlation and standard multiple regressions were used to determine the relationship between personality traits and parental expectations of child development. The analyses gave information about the proportion of the variance in parents' expectations of child's development accounted for by the model, the significance of the model, and the significance of the predictor variable.

\section{Results}

The results of the present study are presented in Table 1 and table 2, showing parents' big five personality as a function of parents expectation on child development. Table 1 indicates that openness to experience had positive correlation, but extraversion, and emotional stability had negative correlation with parental expectation. On the other hand, agreeableness, conscientiousness, had no significant correlation with parental expectation. Both extraversion and openness to experience were the significant predictors of parental expectation which is evident from multiple regression results presented in Table 2 . Thus it can be said result partially supported the hypotheses.

Moreover, adjusted $R^{2}$ values in Table 2 indicate that the model (which includes all big five personality dimensions) was significant and explained $24.1 \%$ variance in parental expectation. Standard $\beta$ value indicates that Openness to experience was the strongest predictor of parental expectation $(\beta=.404, p<.05)$ followed by extraversion $(\beta=$ $-.315, p<.05)$. Part correlation coefficients furthermore indicate that the unique contribution to the explanation of variance in parental expectation was $11.833 \%$ for extraversion, $1.369 \%$ for agreeableness, $1.5129 \%$ for conscientiousness, $1.061 \%$ for emotional stability and $13.69 \%$ for openness to experience. Uniquely they explained total $29.47 \%$ variance in parental expectation which was $5.37 \%$ more than what they explained jointly. Findings like this with that of Table 2 indicate that the interactive influence of Big Five personality on parental expectation was different from the unique influence of a single personality trait on parents' expectation.

Table 1. Bivariate Correlation between Parental Expectation and Big-Five Personality

\begin{tabular}{|l|c|c|c|c|c|c|}
\hline \multicolumn{1}{|c|}{ Variables } & 1 & 2 & 3 & 4 & 5 & 6 \\
\hline 1. Parental expectation & -- & & & & & \\
\hline 2. Extraversion & $-.374^{* *}$ & -- & & & & \\
\hline 3. Agreeableness & -.194 & .116 & -- & & & \\
\hline 4. Conscientiousness & -.143 & -.050 & .081 & -- & & \\
\hline 5. Emotional stability & $-.263^{*}$ & -.109 & .224 & .202 & -- & \\
\hline $\begin{array}{l}\text { 6. Openness to } \\
\text { experience }\end{array}$ & $.432^{* *}$ & .073 & -.193 & -.021 & $-.374^{* * *}$ & -- \\
\hline
\end{tabular}

$* * * \mathrm{p}<.01, * * \mathrm{p}<.05, * \mathrm{p}=.05$

Table 2. Regression of Parental Expectation on Parent's Big-Five Personality

\begin{tabular}{|c|c|c|c|c|c|}
\hline Variables & $\mathrm{B}$ & $\mathrm{SE} \mathrm{B}$ & $\beta$ & $\mathrm{t}$ & $\mathrm{sr}$ \\
\hline Extraversion & -.118 & .048 & -.351 & -2.469 & -.344 \\
\hline Agreeableness & -.015 & .055 & -.039 & -.267 & -.037 \\
\hline Conscientiousness & -.038 & .043 & -.126 & -.882 & -.123 \\
\hline Emotional stability & -.028 & .038 & -.116 & -.740 & -.103 \\
\hline Openness to & .093 & .035 & .404 & 2.655 & .370 \\
\hline
\end{tabular}

Note. Adjusted $R^{2}=.241\left(F_{5,97}=3.475, p<.05\right)$.

\section{Discussion}

The aim of this study was to investigate whether parents' expectations of child's development depended on their Big-five personality traits. Reviewing the relevant literature in detailed, it was hypothesized that, variation in parents' expectations of child development would be predicted by emotional stability, conscientiousness, openness to experience, agreeableness and extraversion. The findings of the study partially supported hypotheses. Results presented in the Table 1, and Table 2 indicated that both extraversion and openness to experience contributed to parental expectation.

Results presented in Table 2 indicate that openness to experience was the strongest predictor and also was the best in explaining the variance in parental expectation. That is, high openness to experience of parents' led to expectation about their child development. This is possibly because Individual who is high on this trait is imaginative, creative, original, and curious. For that, parents' who are highly openness to experience imagine child's future success at higher level. Thus, they expect more from their child. Moreover, Metsapelto and Pulkkinen [29] has found Authoritative parents, who were high in nurturance and high to moderate in parental knowledge, high to moderate in openness to experience. Similarly Authoritarian parents, who were low in nurturance, high to moderate in restrictiveness, and moderate to low in parental knowledge, were low in openness to experience. On the other hand Permissive parents, who were low in restrictiveness and 
parental knowledge and moderate in nurturance, were high in openness to experience. In addition, Research on parenting style, has evidenced that authoritarian and authoritative both style imposed demand on children [25]. Thus it might be possible that, high openness to experience parents' used Authoritative style and imposed demand on children.

This study also indicates a negative correlation between extraversion and parental expectation. This is possibly because extraversion characterized by positive emotions, and the tendency to seek out stimulation and the company of others. According to Costa and McCrae [12] extravert people characterized by gregariousness, is a state describes the quality of a persons' interpersonal relationship. For that, their quality of relationship with children is warmth and they have appropriate knowledge about their child's competence. Since extravert parents' appropriate knowledge about their child's competence, they might make more realistic expectation about their child development. Moreover, extravert parents' are also having positive emotion, warmth, and assertiveness. Therefore, they might have less expectation about their child development. In addition, extraverts maintained level of stimulation and are more aware of rewarding aspect of environment [39]. In that situation, extravert parents' may have less expectation about child development because they do not want to be frustrated. Thus it can be said that extravert parents might function better as a parents than less extensive parents.

Although the present study tried to maintain a sound methodology and analysis of data, nerveless it is not free from certain draw backs and limitations. Following may be said to be the major limitations of the study. The study was conducted on a limited number of respondents. In this study non-probability sampling techniques was also used. In addition socio demographic factors of parents (e.g., parents' education, age sex etc.), others situational factors (e.g., child birth order, number of sibling, child's sex etc.) was not introduced. Therefore, further studies considering these factors are necessary get a better picture on this matter. However, other personality factor such as self-esteem, locus of control of parent's helps to get a better picture on this. Moreover, demographic factors, psychosocial factors, parenting style and personality can be studied combined.

\section{Conclusions}

Findings of the present study shed some light on the positive parenting such as extravert parents' are more positive parents. Thus, in conclusion it can be said that, a better understanding of the antecedents of parental expectations would benefit researchers, child psychologists, counselors, and mental health professionals working in the field of child development. The Understanding of how parental personality is related to their expectations about child's development might help us to formulate positive parenting strategies to promote healthy mental life of children.

\section{Acknowledgements}

Authors would like to express their special thanks to all the parents who voluntarily participated in this study, and who helped to collect the data.

\section{REFERENCES}

[1] K. C. Stoiber, T. G. Hoguhton. The Relationship of adolescence mothers' expectation, knowledge, and beliefs to their young children's coping behavior, Infant Mental Health Journal, vol.14, No.1, 61-79, 1993.

[2] S. T. Azar, D. R. Robinson, E. Hekimian, C. T. Twentyman. Unrealistic expectations and problem-solving ability in maltreating and comparison mothers. Journal of Consulting and Clinical psychology, Vol. 52, 687-691, 1984.

[3] R. Vasta. Physical child abuse: A dual-component analysis, Developmental Review, vol.2, 125-149, 1982.

[4] S. McArdle, J. L. Duda. Exploring the etiology of perfectionism and perceptions of self-worth in young athletes. Social Development, vol.17, No.4, 980-997, 2008.

[5] E. Flouri, D. Hawkes. Ambitious mothers-successful daughters: mothers' early expectations for children's' education and children's earning and sense of control in adult life, British Journal of Educational Psychology, vol.78, No.3, 411-433, 2010. [5]

[6] M.A. Beutel, K. G. Anderson. Race and the educational expectations of parents and children: the case of South Africa, Sociological Quarterl, vol. 49, No. 2, 335-36, 2006.

[7] P. Bourdieu, J. Passeron. Reproduction in education, society, and culture. London, England, Sage, 1977.

[8] R.A. Le Vine. Human parental care: Universal goals, cultural strategies, individual behavior, New York, NY: Willy, 1988.

[9] M. Zahan. Assets, parental expectations and involvement, and children's educational performance, Children and Youth Services Review, vol. 28, 961-975, 2005.

[10] Ryckman. Theories of personality, Belmont, CA, Thomson/Wadsaorth, 2004.

[11] W.A. Goldberg. Marital quality, parental personality, and spousal agreement about perceptions and expectations for children, Merrill-Palmer Quarterly, Journal of Developmental Psychology, vol.36, No.4, 531-556, 1990.

[12] P.T. Costa, R.R Jr McCrae. Four ways five factors are basic, Personality and individual difference, vol.13, 653-665, 1992.

[13] G. Kochanska, A. E. Friesenborg, L.A. Lange, M.M Martel, G. Kochanska. Parents' personality and infants' temperament as contributors to their emerging relationship, Journal of Personality and Social Psychology, vol.86, No.5, 2004.

[14] P. Nancy, Hastie, Reid. Evidence evaluation in complex decision making, Journal of Personality and Social Psychology, vol.5, No.2, 242-258, 1986. 
[15] M. Vokola, L. Tsa. ousis, L. Mikolaou. The role of emotional intelligence and personality on attitudes toward organizational change. Journal of Capital Psychology, vol.19, No.2, 550-567, 1986.

[16] L. Zhang. Dose the big five predict learning approach, Personality and Individual Differences, vol.34, No.8, 1431-1446, 2003

[17] A.K. Kamp. Parent's personality and media performance, The journal of Personify Psychology, vol.20, No.1, 225-234, 2001.

[18] B. Ekehammar, N. Akrami. The relation between personality and prejudices: a variable and person centered approach, European Journal of Personality, vol.17, No.6, 449-464, 2003.

[19] E. Diener, S. Oishi, R. E. Lucas. Personality, culture and subjective wellbeing: emotional and cognitive evaluation of life, Annual Review of Psychology, vol.54, 403-25, 2003.

[20] P.T. Costa, R.R Jr McCrae. Influence of extraversion and neuroticism on subjective well-being: Happy and unhappy people, Journal of Personality and Social Psychology, vol. 38, No. 4, 668-678, 1980.

[21] M. Donnellan, L. Brent, Dannelle, R.D. Conger. Personality, Family History, and Competence in Early Adult Romantic relationship, Journal of Personality and Social Psychology, vol.73, 1141-1180,2005

[22] Y. Beek, M. L. Genta, A. Costabile, A. Sansavini. Maternal expectations about infant development of pre-term and full-term infants: a cross-national comparison, Infant and Child Development, vol.15, No.1, 41-58, 2006.

[23] S.B. Silverberg, M.S. Marczak, D. M. Gondoli. Maternal depressive symptoms and achievement-related outcomes among adolescents' daughters: Variations by family structure, Journal of Early Adolescence, vol.16, 90-109, 1996.

[24] E. Kortlander, P.C. Kendall, S.M. Panichelli-Mindel Maternal expectations and attributions about coping in anxious children, Journal of Anxiety Disorders, vol.11, 297-315, 1997

[25] D. E. Papalia, S.W. Olds, R.D. Feldman. Human development, Boston, Mc Grage-Hill, 1988.

[26] G. Kochanska, A. E. Friesenborg, L.A. Lange, M.M. Martel. Parents' personality and infants' temperament as contributors to their emerging relationship, Journal of Personality and Social Psychology, vol.86, No.5, 744-759, 2004.
[27] R. Levy-Shiff, R. Israelashvili. Antecedents of fathering: Some further exploration, Developmental Psychology, vol.24, 434-440, 1988.

[28] S. H. Losoya, S. Callor, D.C. Rowe, H. H Goldsmith. Origins of familial similarity in parenting: A study of twins and adoptive siblings, Developmental Psychology, vol.33, 10121023, 1997.

[29] R. Metsapelto, L. Pulkkinen. Personality traits and parenting: Neuroticism, Extraversion, and Openness to Experience as discriminative factors, European Journal of Personality, vol.17, 59-78, 2003.

[30] L.A.Clark, G. Kochanska, R. Ready. Mothers' personality and It's interaction with child temperament as predictors of parenting behavior, Journal of Personality and Social Psychology, vol. 7, 274-285, 2000.

[31] D. Lee-Baggley, M.Preece, A. DeLongis. Coping with interpersonal stress: Role of big, five traits, Journal of Personality, vol.73, 1141-1180, 2005.

[32] J. Belsky, N. Barends. Personality and parenting, Mahwah, NJ, Erlbaum, 2002.

[33] E.M. Cummings, P.T. Davies. Maternal depression and child development, Journal of Child Psychology and Psychiatry, vol.35, 73-112, 1994.

[34] S.H. Goodman, I.H. Gotlib. Risk for psychopathology in the Children of depressed parents: A developmental model for understanding mechanisms of transmission. Psychological Review, vol.106, 458-490, 1970.

[35] A. Huque. Bangle version (Huque, 2008) of Saucer's (1994) Big Five- Mini Markers, Unpublished manuscript, Dhaka, 2008.

[36] F.S. Mayer, K. Sulton. Personality: An integrative approach, New Jersey, UK, Prentice Hall Incc, 1996.

[37] G. Saucer. Mini Markers: A brief version of Goldberg's unipolar big-five markers, Journal of Personality Assessment, vol.63, No.3, 506-516, 1994.

[38] J.K. Palmer, J.M. Loveland. Further investigation of the psychometric properties of Saucer's Big Five 'Mini-Markers:' Evidence for criterion and construct validity. Individual Difference Research, vol.2, No.3, 231-238, 2004.

[39] M.E. Mullins, J.M. Polson, T. Lanch, K. Kehoc. Respondent perceptions of integrity and personality measures: Does response format make a difference? Applied H.R.M. Research, vol.11, No.2, 2007. 